\title{
Abrogation of STAT3 activation cascade by Ginkgolide C mitigates tumorigenesis in lung cancer preclinical model
}

\author{
Min Hee Yang ${ }^{1}$, Jae-Young $\mathrm{Um}^{2}$, Gautam Sethi ${ }^{3}$, and Kwang Seok $\mathrm{AHn}^{1}$ \\ ${ }^{1}$ Kyung Hee Univ. \\ ${ }^{2}$ Kyung Hee University \\ ${ }^{3}$ Yong Loo Lin School of Medicine
}

August 3, 2020

\begin{abstract}
Background and purpose: Ginkgolide C (GGC) isolated form Ginko biloba (Ginkgoaceae) leaf can demonstrate pleiotropic pharmacological actions although. its anti-oncogenic impact in non-small cell lung cancer (NSCLC) model has not been reconnoitered. As signal transducer and activator of transcription 3 (STAT3) cascade can promote tumor growth and survival, we contemplated that GGC may interrupt this signaling cascade to expend its anti-cancer actions in NSCLC. Experimental approach: The effect of GGC on STAT3 activation, associated protein kinases, STAT3-regulated gene products, cellular proliferation, and apoptosis was examined. The in vivo effect of GGC on the growth of human NSCLC xenograft tumors in athymic $\mathrm{nu} / \mathrm{nu}$ female mice was also investigated. Key results: GGC attenuated the phosphorylation of STAT3 and varying upstream kinases effectively. Exposure to pervanadate modulated GGC-induced down-regulation of STAT3 activation and promoted an elevation in the level of PTP? protein. Indeed, silencing of the PTP??gene reversed the GGC-promoted abrogation of STAT3 activation and apoptosis. Moreover, GGC exposure significantly reduced NSCLC tumor growth without demonstrating significant adverse effects via decreasing levels of p-STAT3 in mice tissues. Conclusions and Implications: Overall, the findings support that GGC may exhibit anti-neoplastic actions by mitigation of STAT3 signaling cascade in NSCLC.
\end{abstract}

\section{Abrogation of STAT3 activation cascade by Ginkgolide C mitigates tumorigenesis in lung cancer preclinical model}

Min Hee Yang ${ }^{1,2}$, Jae-Young $\mathrm{Um}^{2}$, Gautam Sethi ${ }^{3,}$, Kwang Seok $\mathrm{Ahn}^{1,2^{*}}$

${ }^{1}$ KHU-KIST Department of Converging Science and Technology, Kyung Hee University, Seoul 02447, Republic of Korea

${ }^{2}$ Department of Science in Korean Medicine, Kyung Hee University, 24 Kyungheedae-ro, Dongdaemun-gu, Seoul 02447, Republic of Korea

${ }^{3}$ Department of Pharmacology, Yong Loo Lin School of Medicine, National University of Singapore, Singapore 117600, Singapore

*Address correspondence to:

Dr. Kwang Seok Ahn, Department of Science in Korean Medicine, College of Korean Medicine, Kyung Hee University, 24 Kyungheedae-ro, Dongdaemun-gu, Seoul 02447, Republic of Korea. Tel: (+82) 2-961-2316; Email: ksahn@khu.ac.kr

Dr. Gautam Sethi, Department of Pharmacology, Yong Loo Lin School of Medicine, National University of Singapore, Singapore 117600, Singapore. Email: phcgs@nus.edu.sg 
Running Title: GGC negates carcinogenesis in NSCLC.

\section{Summary}

Background and purpose: Ginkgolide C (GGC) isolated form Ginko biloba (Ginkgoaceae) leaf can demonstrate pleiotropic pharmacological actions although. its anti-oncogenic impact in non-small cell lung cancer (NSCLC) model has not been reconnoitered. As signal transducer and activator of transcription 3 (STAT3) cascade can promote tumor growth and survival, we contemplated that GGC may interrupt this signaling cascade to expend its anti-cancer actions in NSCLC.

Experimental approach: The effect of GGC on STAT3 activation, associated protein kinases, STAT3regulated gene products, cellular proliferation, and apoptosis was examined. The in vivo effect of GGC on the growth of human NSCLC xenograft tumors in athymic $n u / n u$ female mice was also investigated.

Key results: GGC attenuated the phosphorylation of STAT3 and varying upstream kinases effectively. Exposure to pervanadate modulated GGC-induced down-regulation of STAT3 activation and promoted an

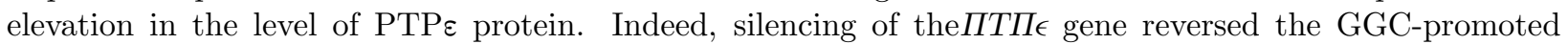
abrogation of STAT3 activation and apoptosis. Moreover, GGC exposure significantly reduced NSCLC tumor growth without demonstrating significant adverse effects via decreasing levels of p-STAT3 in mice tissues.

Conclusions and Implications: Overall, the findings support that GGC may exhibit anti-neoplastic actions by mitigation of STAT3 signaling cascade in NSCLC.

Keywords: Ginkgolide C, NSCLC, STAT3, xenograft, PTPع.

\section{Introduction}

Lung cancer contributes largely to cancer-related mortality worldwide (Huang et al., 2016; Lee, Kim, Sethi \& Ahn, 2015; Mason, 1949; Siegel, Miller \& Jemal, 2015). Human lung cancer consists of non-small lung cancer (NSCLC) and small cell lung cancer (SCLC) (Zhang et al., 2016; Zhu et al., 2018). NSCLC is more common form and five-year survival rate is generally less than $5 \%$ in advanced cases (Lee et al., 2019a; Reck, Heigener, Mok, Soria \& Rabe, 2013; Wang et al., 2018; Yang, Lee, Ko, Jung, Sethi \& Ahn, 2019). Surgery operation, radiation therapy, chemotherapy, targeted therapy etc are are routinely used for management of NSCLC patients (Lee, Chinnathambi, Alharbi, Shair, Sethi \& Ahn, 2019; Lee, Kim, Lee, Sethi \& Ahn, 2018b; Yang et al., 2005). However, these traditional treatment modalities may be associated with low specificity and serious side effects on patients (Artal Cortes, Calera Urquizu \& Hernando Cubero, 2015; Ko, Nam, Um, Jung, Sethi \& Ahn, 2018; Lee et al., 2018; Liu, Kuang, Wu, Jin \& Sun, 2016). Hence, the evolution of novel agents for the NSCLC therapy are still required.

Signal transducer and activators of transcription (STAT) family is made up of STAT1, STAT2, STAT3, STAT4, STAT5 $\alpha$, STAT5 $\beta$, and STAT6 (Ashrafizadeh et al., 2019; Loh, Arya, Naema, Wong, Sethi \& Looi, 2019; Mohan et al., 2020; Schindler, Levy \& Decker, 2007; Wong, Hirpara, Pervaiz, Eu, Sethi \& Goh, 2017; Yu, Pardoll \& Jove, 2009). Among these proteins, STAT3 activated has been found to be deregulated in varied human cancer cells, such as head and neck, lung, breast, prostate, kidney, pancreas, liver cancer lymphomas, multiple myeloma (Arora, Kumar, Arfuso, Chng \& Sethi, 2018; Hwang et al., 2019; Kim et al., 2014; Lee et al., 2014; Lee, Kim, Lee, Um, Sethi \& Ahn, 2019; Lee et al., 2019b; Loh, Arya, Naema, Wong, Sethi \& Looi, 2019; Yang et al., 2019). STAT3 can regulate varying hallmarks of cancer and promote expression of various tumorigenic genes (Baek et al., 2017; Kim et al., 2018; Lee et al., 2017; Lee, Kim, Kim, Sethi \& Ahn, 2015; Shanmugam et al., 2015). The activation of STAT3 can be mediated by upstream kinases (JAK1/2 and Src) and undergo dimerization, translocation into nucleus, DNA binding, thus promoting transcription (Baek et al., 2016a; Fathi, Rashidi, Khodadadi, Shahi \& Sharifi, 2018; Lee, Kim, Lee, Sethi \& Ahn, 2018a; Wang \& Sun, 2014). In addition, diverse protein tyrosine phosphatases (PTPs) such as SHP-1, SHP-2, PTEN, and

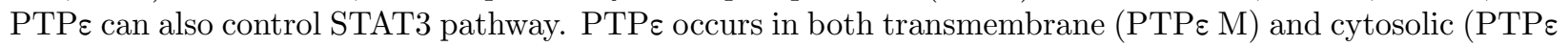
C) forms (Nakamura, Mizuno \& Kikuchi, 1996; Yang et al., 2019). PTPع M can be expressed in brain, testis, and lung, whereas PTP $\varepsilon$ C is expressed in spleen thymus, and peritoneal macrophages (Elson \& Leder, 1995; 
Tanuma, Nakamura \& Kikuchi, 1999; Tanuma, Shima, Nakamura \& Kikuchi, 2001). Interestingly, PTPع C was found to abrogate IL-6-stimulated JAK/STAT casacde in murine leukemia cells (Tanuma, Nakamura, Shima \& Kikuchi, 2000).

Natural products remain the mainstay for discovery of novel anti-cancer drugs (Dai et al., 2015; Shanmugam, Warrier, Kumar, Sethi \& Arfuso, 2017; Siveen et al., 2014; Tewari et al., 2018; Zhou et al., 2020). Ginkgo biloba (Ginkgoaceae) is one of the common ancient trees, and has been used as a medical herb for a long time in clinical therapy in oriental and western medicine (Yeh, Shou, Lin, Chen, Chiang \& Yeh, 2015). Ginkgolide C (GGC) isolated from Ginkgo bilobaleaves, is a flavone with multiple biological functions (Huang et al., 2014; Huang, Chen, Liu, Wu \& Liou, 2018; Liou, Lai, Chen, Wang, Wei \& Huang, 2015; Zhang et al., 2018). GGC has been implicated to increase lipolysis and suppress adipogenesis in 3T3-L1 adipocyte through modulation of AMPK signaling cascades (Liou, Lai, Chen, Wang, Wei \& Huang, 2015). In addition, GGC can alleviate myocardial ischemia/reperfusion injury through suppression of CD40-NF-kB signaling pathway (Zhang et al., 2018). GGC also has been reported to reduce oleic acid-induced lipid accumulation via the Sirt1/AMPK pathway (Huang, Chen, Liu, Wu \& Liou, 2018). However, up to now, there is little research on the impact of GGC on the progression of human cancers. STAT3 has been implicated to regulate tumorigeensis (Dai et al., 2016; Li et al., 2013), it may be possible that GGC may exert its anti-cancer actions through affecting this pathway.

In this study, we investigated whether GGC induce apoptosis and affect NSCLC growth by modifying the activation of STAT3 pathway. We noted that GGC can mitigate STAT3 activation by affecting PTP $\varepsilon$ tyrosine phosphatase in NSCLC cells that mediates its anti-oncogenic impact. Thus, GGC can act as a potent blocker of STAT3 signaling pathway and abrogate tumor growth effectively in NSCLC model.

\section{Materials and Methods}

Reagents and cell lines

Ginkgolide C (GGC) was purchased from Weikeqi Biological Technology (Chengdu, Sichuan. China). 3(4,5-dimethylthiazol-2-yl)-2,5-diphenyltetrazolium bromide (MTT) and bovine serum albumin (BSA) were purchased from Sigma-Aldrich (St. Louis, MO). Human lung carcinoma cells (A549 and H1299 cells) were obtained from the American Type Culture Collection (Manassas, VA, USA). A549 cells were grown in DMEM low glucose. H1299 cells was cultured in RPMI 1640 medium and maintained at 37 under 5\% $\mathrm{CO}_{2}$ atmosphere.

MTT assay

Cell viability was measured using an MTT assay as described earlier (Lee et al., 2019b).

Western blot analysis

After treating with GGC for indicated concentrations and time points, western blotting was executed as elaborated previously (Lee et al., 2019b).

EMSA for STAT3-DNA binding

STAT3-DNA binding was analyzed by electrophoretic mobility shift assay (EMSA) as elaborated previously (Lee et al., 2019b).

Immunocytochemistry

A549 and H1299 cells were treated with GGC $30 \mu \mathrm{M}$ for $12 \mathrm{~h}$ and thereafter immunocytochemistry was done (Lee et al., 2019b).

Transfection with PTP $\varepsilon$ siRNA

To inhibit PTP $\varepsilon$ expression by RNA interference, A549 cells were transfected with 50 nM STAT3 siRNA or $100 \mathrm{nM}$ scrambled siRNA using NEON Transfection system (Invitrogen). Then cells were incubated in $10 \%$ FBS-supplemented DMEM low glucose medium for $24 \mathrm{~h}$. 
Transfection of plasmids

To establish the link between anti-cancer effects of GC and involvement of STAT3 signaling pathway, A549 cells were transfected with STAT3-C Flag pRc/CMV containing plasmid DNA. Cells $\left(5 \times 10^{4}\right.$ cells/well) were transfected with STAT3C DNA (300 ng/well) in serum-free media for $48 \mathrm{~h}$ by iN-fect transfection Reagent. After $48 \mathrm{~h}$, cells were washed with 1x PBS and treated with GGC $(30 \mu \mathrm{M})$ in complete media for $24 \mathrm{~h}$.

Cell cycle analysis

Cell cycle analysis was performed to examine the effects of GGC on cell cycle progression as described before (Lee et al., 2019c).

Annexin $\mathrm{V}$ and TUNEL assays

Annexin V assay was performed to determined apoptosis in A549 and H1299 cells as elaborated upon previously (Lee et al., 2019b).

Real-time cell proliferation analysis

Cell growth behavior was performed using the Roche xCELLigence Real-Time Cell Analyzer (RTCA) as reported previously (Lee et al., 2019c).

Reverse transcription polymerase chain reaction (RT-PCR)

A549 cells were treated with GGC $30 \mu \mathrm{M}$ for $24 \mathrm{~h}$ and H1299 cells were pre-treated with GGC $30 \mu \mathrm{M}$ for 12 $\mathrm{h}$ and treated with IL-6 $(20 \mathrm{ng} / \mathrm{ml})$ for $24 \mathrm{~h}$. Total RNA was extracted and RT-PCR was done.

Experimental Protocol

All procedures involving animals were reviewed and approved by Kyung Hee University Institutional Animal Care and Use committee [KHUASP(SE)-18-124]. A549 xenograft model was established and athymic nu/nu female mice were randomized into following four different treatment groups ( $n=6 /$ group). Tumor volumes were measured by Digimatic caliper every 5 days and mice body weight was measured about 2 or 3 day intervals. Mice were killed 5 days later after last therapy and tumor tissues were further processed as described before (Jung et al., 2019)

Western blot analysis of tumor tissues

Lung tumor tissues were subjected to western blotting as described earlier (Jung et al., 2019).

Immunohistochemical study

Immunohistochemistry was done as elaborated previously (Jung et al., 2019).

Statistical analysis

All experiments are presented as the mean \pm standard deviation (SD). Statistical significance of the data compared with the non-treated control was determined using the Mann-Whitney U test. Significance was set at $P<0.05$.

\section{Results}

\section{GGC exhibited cytotoxic activity against lung cancer cells.}

First, we found that GGC mitigated the viability of A549 and H1299 cells significantly in dose response studies (Fig. 1B). Interestingly, it demonstrated lower cytotoxicity against HEL 299 normal lung cells as compared to tumor cells.

\section{GGC alters STAT3 phosphorylation substantially.}

We examined the impact of GGC on STAT3 activation using western blotting (Fig. 1C). We noted that GGC suppressed the phosphorylation of STAT3 (Tyr 705), but had minimal impact on STAT5 activation 
(Fig. 1G). We next determined whether GGC can modulate DNA binding property of STAT3. As shown in Fig. 1D, GGC can effectively reduce DNA binding ability as well as attenuate STAT3 translocation from the cytoplasm to nucleus in A549 cells (Fig.1E).

\section{GGC represses activation of kinases controlling STAT3 phosphorylation.}

As STAT3 can be activated by upstream kinases, we deciphered the actions of GGC on the phosphorylation of JAK1, JAK2, and Src. GGC inhibited p-JAK1, p-JAK2, and p-Src levels (Fig. 1F) without altering total JAK1, JAK2, and Src proteins. We also found that IL-6 promoted phosphorylation of STAT3 was maximally induced at 15 min after IL-6 treatment (Fig. 2A). As shown Fig. 2B and 2C, GGC attenuated IL-6-driven STAT3 phosphorylation and upstream signaling proteins in time dependent manner. In addition, H1299 cells were transfected with STAT3-luciferase construct and when the cells exposed to GGC prior to stimulation, IL-6-induced STAT3 activity was repressed significantly.

\section{Overexpression of STAT3 relieves GGC-promoted apoptosis and reduction in viability.}

In addition, to confirm whether the mitigation of STAT3 activation was crucial in GGC-driven apoptosis, A549 cells were transfected with a STAT3C-expressing construct followed by GGC exposure. As shown Fig. 2H, STAT3C-overexpression remarkably increased p-STAT3(Tyr705) and STAT3 levels. Moreover, this increase led to a substantial decrease in GGC-regulated apoptosis and its effect on viability as compared with control cells (Fig. 1F and Fig.4F).

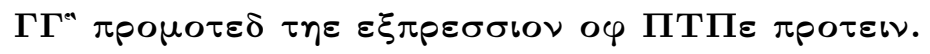

To study the mechanism of GGC-mediated inhibition of STAT3 phosphorylation, we elucidated the impact of GGC by modulating of protein tyrosine phosphatase. Pretraetment with sodium pervanadate abrogated reduction in p-STAT3 levels caused by GGC (Fig. 2E). Moreover, we noted that GGC led to a dramatic

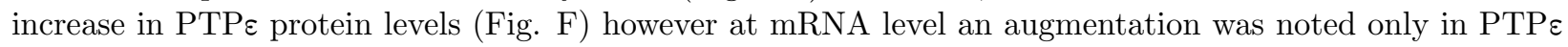

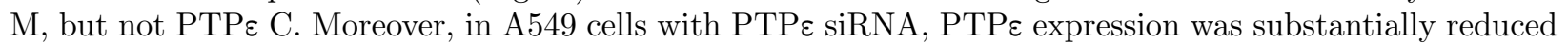
((Fig. 2H) and GGC was unable to affect STAT3 phosphorylation in PTP $\varepsilon$ knockdown cells (Fig. 2I). In addition, PTP $\varepsilon$ knockdown attenuated GGC-induced PARP cleavage suggesting that this phosphatase may have a vital role in regulating anti-cancer properties of GCC.

\section{GGC induces apoptotic cell death in NSCLC.}

To analyze the apoptotic effect of GGC, we performed various assays to measure programmed cell death. As shown Fig. 3A, GGC caused subG1 phase arrest in A549 and H1299 cells. Early apoptosis was promoted and peak was shift to right side with apoptosis. In addition, as shown in Fig. 3B, GGC promoted apoptosis through modulating caspase-3 and PARP cleavage. Next, we noted that cell proliferation was significantly reduced upon GGC exposure in both A549 and H1299 cells (Fig. 3C). Moreover, GGC also attenuated the expression of various oncogenic molecules at protein and mRNA levels (Fig. 4A and C). Moreover, IL-6 promoted articulation of oncogenic markers was also affected upon GGC exposure (Fig. 4B and D).

\section{GGC exerts antitumor effects in preclinical model.}

We examined the impact of GGC to modulate tumor growth in xenograft model as per the protocol specified in Fig. 5A. The results suggested that in control group I, tumor volume sharply increased but markedly decreased in groups II and III (Fig. 5C). Moreover, tumor size and weight were attenuated in groups II, and III as compared to control (Fig. 5B and D), without altering the body weight (Fig. 5E).

\section{GGC alters levels of various oncogenic markers.}

First as measured by immunohistochemical analysis it found that GGC treatment caused a marked downregulation in the expression of p-STAT3, Ki-67, and VEGF proteins (Fig. 6A). Thereafter, as shown Fig. 6B, GGC increased PTP $\varepsilon$ protein level but decreased the levels of phopho-STAT3 and different phosphorylated kinases as measured by western blotting. Next, cleaved caspase-3 and PARP levels were significantly elevated 
in tissues harvested from GCC exposed mice (Fig. 6D). Finally, GGC reduced the expression of diverse oncogenic proteins that can regulate tumorigenesis in NSCLC model (Fig. 5E).

\section{Discussion}

In traditional Chinese medicine, Ginkgo leaf extract is widely used as medical herb to treat cardiovascular disorders (Xu, Hu, Shen \& McQuillan, 2015). It has been reported that GGC isolated from Ginkgo leaf , can exhibit diverse pharmacological properties (Huang et al., 2014; Huang, Chen, Liu, Wu \& Liou, 2018; Liou, Lai, Chen, Wang, Wei \& Huang, 2015; Zhang et al., 2018). This is the first investigation that has analyzed the potential impact of GGC and its mechanism in NSCLC model. We found that GGC diminished activation of STAT3 as well as that of JAK1, JAK2, and c-Src phosphorylation. This inhibition led to an induction in the expression of phosphatase PTPE. GGC also mitigated proliferation and promoted apoptosis through down-regulating STAT3 signaling cascade. Moreover, it attenuated tumor growth and survival in a xenograft mouse model without displaying any toxicity.

Aberrant STAT3 activation has been associated with various tumors, including head and neck, lung, breast, prostate, kidney, pancreas, liver cancer, lymphomas, and multiple myeloma (Banerjee \& Resat, 2016; Bharti, Donato \& Aggarwal, 2003; Chai et al., 2016; Hwang et al., 2019; Jung et al., 2018). We first investigated if GGC could alter constitutive and inducible STAT3 phosphorylation in NSCLC cells. We noted that GGC abrogated STAT3 phosphorylation and localization of this protein into the nucleus. Phosphorylation of JAKs has been linked to STAT3 activation (Ahn, Sethi, Sung, Goel, Ralhan \& Aggarwal, 2008; Baek et al., 2017; Bowman, Garcia, Turkson \& Jove, 2000). Interestingly, GGC mitigated JAK1, JAK2, as well as c-Src phosphorylation substantially and overexpression of STAT3 not only alleviated the inhibitory actions of GGC on p-STAT3 levels but also relieved apoptosis caused by this agent.

Recently, many studies have reported that protein tyrosine phosphatases (PTPs) may negatively control STAT3 signaling pathway (Ahn, Sethi, Sung, Goel, Ralhan \& Aggarwal, 2008; Baek et al., 2016a; Baek et al., 2016b; Kim, Morales, Jang, Cho \& Kim, 2018). We noted that GGC may have altered STAT3 activation by causing the modulation of PTPs. Interestingly, GGC promoted PTP $\varepsilon$ expression at protein and mRNA levels. Moreover, GGC could increase PTP $\varepsilon$ M expression also at mRNA levels. Moreover, the silencing of PTPE expression repudiated GGC-driven attenuation of STAT3 activation and induction of apoptosis. However, how GCC can stimulate PTP $\varepsilon$ levels and if GCC can also alter, its activity needs additional investigations.

It has been reported that reduction of STAT3 activity may decrease the survival ability of tumor cells (Aoki, Feldman \& Tosato, 2003). Hence, we observed if GGC could alter proliferation and promote apoptosis in NSCLC cells. GGC induced apoptotic death was detected by cell cycle, annexin V and TUNEL assays. We demonstrated that GGC caused sub-G1 arrest, early apoptosis, and mediated the activation of caspase-3 and PARP. In addition, we noted that GGC dramatically reduced the expression of oncogenic molecules such as anti-apoptotic and metastasis promoting proteins that may contribute to its varying anti-neoplastic activities.

In a NSCLC preclinical model, GGC significantly suppressed lung tumor growth, altered the levels of pSTAT3, PTP $\varepsilon$, and caspase-3 in treated groups. These modifications are consonant with its observed in vitro actions and were noted in the absence of any adverse effects. Thus, our preclinical studies imply that GGC may have a potential as a novel therapeutic agent for the management of NSCLC.

In summary, it was noticed that GGC can exert pleiotropic anti-neoplastic effects through modulating STAT3 signaling pathway by affecting the PTP $\varepsilon$ expression. Taken together, GGC may act as an effective inhibitor of STAT3 phosphorylation and its function can be investigated further in alleviation of different malignancies.

Abbreviations
GGC: Ginkgolide C;
STAT3: signal transducer and activator of transcription 3; 
c/w : Cell per well;

DAPI: 4',6-Diamidino-2-Phenylindole, Dihydrochloride;

FBS: Fetal bovine serum;

GAPDH: Glyceraldehyde 3-phosphate dehydrogenase;

HRP: Horseradish peroxidase;

IHC: Immunohistochemistry;

ip: Intraperitoneal injection;

MMP: Matrix metalloproteinase;

DMEM: Dulbecco's Modified Eagle Medium;

NSCLC: Non-small cell lung carcinoma;

NT : Non treat;

P/S: Penicillin-streptomycin;

RTCA: Real-time cell analysis;

RT-PCR: Reverse transcription polymerase chain reaction;

SFM: Serum free media;

PTP: Protein tyrosine phosphatase;

JAK: Janus kinase;

PARP: Poly (ADP-ribose) polymerase;

VEGF: vascular endothelial growth factor

Acknowledgement

This work was supported by a National Research Foundation of Korea (NRF) grant funded by the Korean government (MSIP) (NRF-2017M3A9E4065333 and 2018R1D1A1B07042969).

\section{Author's Contributions:}

MHY conducted all the experiments. JYU analyzed the data. G.S. and K.S.A. supervised and wrote the manuscript.

\section{Declaration of competing interest}

The authors have no conflicts of interest to declare.

\section{References}

Ahn KS, Sethi G, Sung B, Goel A, Ralhan R, \& Aggarwal BB (2008). Guggulsterone, a farnesoid X receptor antagonist, inhibits constitutive and inducible STAT3 activation through induction of a protein tyrosine phosphatase SHP-1. Cancer research 68: 4406-4415.

Aoki Y, Feldman GM, \& Tosato G (2003). Inhibition of STAT3 signaling induces apoptosis and decreases survivin expression in primary effusion lymphoma. Blood 101:1535-1542.

Arora L, Kumar AP, Arfuso F, Chng WJ, \& Sethi G (2018). The Role of Signal Transducer and Activator of Transcription 3 (STAT3) and Its Targeted Inhibition in Hematological Malignancies. Cancers 10.

Artal Cortes A, Calera Urquizu L, \& Hernando Cubero J (2015). Adjuvant chemotherapy in non-small cell lung cancer: state-of-the-art. Translational lung cancer research 4:191-197. 
Ashrafizadeh M, Ahmadi Z, Kotla NG, Afshar EG, Samarghandian S, Mandegary A, et al. (2019). Nanoparticles Targeting STATs in Cancer Therapy. Cells 8.

Baek SH, Ko JH, Lee H, Jung J, Kong M, Lee JW, et al. (2016a). Resveratrol inhibits STAT3 signaling pathway through the induction of SOCS-1: Role in apoptosis induction and radiosensitization in head and neck tumor cells. Phytomedicine : international journal of phytotherapy and phytopharmacology 23:566-577.

Baek SH, Lee JH, Kim C, Ko JH, Ryu SH, Lee SG, et al. (2017). Ginkgolic Acid C 17:1, Derived from Ginkgo biloba Leaves, Suppresses Constitutive and Inducible STAT3 Activation through Induction of PTEN and SHP-1 Tyrosine Phosphatase. Molecules 22.

Baek SH, Lee JH, Ko JH, Lee H, Nam D, Lee SG, et al. (2016b). Ginkgetin Blocks Constitutive STAT3 Activation and Induces Apoptosis through Induction of SHP-1 and PTEN Tyrosine Phosphatases. Phytotherapy research : PTR 30: 567-576.

Banerjee K, \& Resat H (2016). Constitutive activation of STAT3 in breast cancer cells: A review. International journal of cancer 138: 2570-2578.

Bharti AC, Donato N, \& Aggarwal BB (2003). Curcumin (diferuloylmethane) inhibits constitutive and IL-6inducible STAT3 phosphorylation in human multiple myeloma cells. Journal of immunology 171: 3863-3871.

Bowman T, Garcia R, Turkson J, \& Jove R (2000). STATs in oncogenesis. Oncogene 19: 2474-2488.

Chai EZ, Shanmugam MK, Arfuso F, Dharmarajan A, Wang C, Kumar AP, et al. (2016). Targeting transcription factor STAT3 for cancer prevention and therapy. Pharmacology \& therapeutics 162: 86-97.

Dai X, Ahn KS, Wang LZ, Kim C, Deivasigamni A, Arfuso F, et al. (2016). Ascochlorin Enhances the Sensitivity of Doxorubicin Leading to the Reversal of Epithelial-to-Mesenchymal Transition in Hepatocellular Carcinoma. Molecular cancer therapeutics 15: 2966-2976.

Dai X, Zhang J, Arfuso F, Chinnathambi A, Zayed ME, Alharbi SA, et al. (2015). Targeting TNF-related apoptosis-inducing ligand (TRAIL) receptor by natural products as a potential therapeutic approach for cancer therapy. Experimental biology and medicine 240: 760-773.

Elson A, \& Leder P (1995). Identification of a cytoplasmic, phorbol ester-inducible isoform of protein tyrosine phosphatase epsilon. Proceedings of the National Academy of Sciences of the United States of America 92:12235-12239.

Fathi N, Rashidi G, Khodadadi A, Shahi S, \& Sharifi S (2018). STAT3 and apoptosis challenges in cancer. International journal of biological macromolecules 117:993-1001.

Huang P, Zhang L, Chai C, Qian XC, Li W, Li JS, et al. (2014). Effects of food and gender on the pharmacokinetics of ginkgolides A, B, C and bilobalide in rats after oral dosing with ginkgo terpene lactones extract. Journal of pharmaceutical and biomedical analysis 100: 138-144.

Huang Q, Li L, Li L, Chen H, Dang Y, Zhang J, et al. (2016). MDM2 knockdown mediated by a triazinemodified dendrimer in the treatment of non-small cell lung cancer. Oncotarget 7: 44013-44022.

Huang WC, Chen YL, Liu HC, Wu SJ, \& Liou CJ (2018). Ginkgolide C reduced oleic acid-induced lipid accumulation in HepG2 cells. Saudi pharmaceutical journal : SPJ : the official publication of the Saudi Pharmaceutical Society 26:1178-1184.

Hwang ST, Kim C, Lee JH, Chinnathambi A, Alharbi SA, Shair OHM, et al. (2019). Cycloastragenol can negate constitutive STAT3 activation and promote paclitaxel-induced apoptosis in human gastric cancer cells. Phytomedicine : international journal of phytotherapy and phytopharmacology 59: 152907.

Jung YY, Lee JH, Nam D, Narula AS, Namjoshi OA, Blough BE, et al. (2018). Anti-myeloma Effects of Icariin Are Mediated Through the Attenuation of JAK/STAT3-Dependent Signaling Cascade. Frontiers in pharmacology 9: 531. 
Jung YY, Shanmugam MK, Narula AS, Kim C, Lee JH, Namjoshi OA, et al. (2019). Oxymatrine Attenuates Tumor Growth and Deactivates STAT5 Signaling in a Lung Cancer Xenograft Model. Cancers 11: 49.

Kim C, Cho SK, Kapoor S, Kumar A, Vali S, Abbasi T, et al. (2014). beta-Caryophyllene oxide inhibits constitutive and inducible STAT3 signaling pathway through induction of the SHP-1 protein tyrosine phosphatase. Molecular carcinogenesis 53: 793-806.

Kim C, Lee SG, Yang WM, Arfuso F, Um JY, Kumar AP, et al. (2018). Formononetin-induced oxidative stress abrogates the activation of STAT3/5 signaling axis and suppresses the tumor growth in multiple myeloma preclinical model. Cancer letters 431: 123-141.

Kim M, Morales LD, Jang IS, Cho YY, \& Kim DJ (2018). Protein Tyrosine Phosphatases as Potential Regulators of STAT3 Signaling. International journal of molecular sciences 19.

Ko JH, Nam D, Um JY, Jung SH, Sethi G, \& Ahn KS (2018). Bergamottin Suppresses Metastasis of Lung Cancer Cells through Abrogation of Diverse Oncogenic Signaling Cascades and Epithelial-to-Mesenchymal Transition. Molecules 23: 1601.

Lee JH, Chiang SY, Nam D, Chung WS, Lee J, Na YS, et al. (2014). Capillarisin inhibits constitutive and inducible STAT3 activation through induction of SHP-1 and SHP-2 tyrosine phosphatases. Cancer letters 345: $140-148$.

Lee JH, Chinnathambi A, Alharbi SA, Shair OHM, Sethi G, \& Ahn KS (2019). Farnesol abrogates epithelial to mesenchymal transition process through regulating Akt/mTOR pathway. Pharmacological research 150: 104504 .

Lee JH, Kim C, Baek SH, Ko JH, Lee SG, Yang WM, et al. (2017). Capsazepine inhibits JAK/STAT3 signaling, tumor growth, and cell survival in prostate cancer. Oncotarget 8: 17700-17711.

Lee JH, Kim C, Kim SH, Sethi G, \& Ahn KS (2015). Farnesol inhibits tumor growth and enhances the anticancer effects of bortezomib in multiple myeloma xenograft mouse model through the modulation of STAT3 signaling pathway. Cancer letters 360: 280-293.

Lee JH, Kim C, Lee J, Um JY, Sethi G, \& Ahn KS (2019). Arctiin is a pharmacological inhibitor of STAT3 phosphorylation at tyrosine 705 residue and potentiates bortezomib-induced apoptotic and antiangiogenic effects in human multiple myeloma cells. Phytomedicine : international journal of phytotherapy and phytopharmacology 55: 282-292.

Lee JH, Kim C, Lee SG, Sethi G, \& Ahn KS (2018a). Ophiopogonin D, a Steroidal Glycoside Abrogates STAT3 Signaling Cascade and Exhibits Anti-Cancer Activity by Causing GSH/GSSG Imbalance in Lung Carcinoma. Cancers 10: 427.

Lee JH, Kim C, Lee SG, Sethi G, \& Ahn KS (2018b). Ophiopogonin D, a Steroidal Glycoside Abrogates STAT3 Signaling Cascade and Exhibits Anti-Cancer Activity by Causing GSH/GSSG Imbalance in Lung Carcinoma. Cancers 10.

Lee JH, Kim C, Lee SG, Yang WM, Um JY, Sethi G, et al. (2018). Ophiopogonin D modulates multiple oncogenic signaling pathways, leading to suppression of proliferation and chemosensitization of human lung cancer cells. Phytomedicine : international journal of phytotherapy and phytopharmacology 40:165-175.

Lee JH, Kim C, Sethi G, \& Ahn KS (2015). Brassinin inhibits STAT3 signaling pathway through modulation of PIAS-3 and SOCS-3 expression and sensitizes human lung cancer xenograft in nude mice to paclitaxel. Oncotarget 6: 6386-6405.

Lee JH, Mohan CD, Basappa S, Rangappa S, Chinnathambi A, Alahmadi TA, et al. (2019a). The IkappaB Kinase Inhibitor ACHP Targets the STAT3 Signaling Pathway in Human Non-Small Cell Lung Carcinoma Cells. Biomolecules 9: 875 . 
Lee JH, Mohan CD, Basappa S, Rangappa S, Chinnathambi A, Alahmadi TA, et al. (2019b). The IkappaB Kinase Inhibitor ACHP Targets the STAT3 Signaling Pathway in Human Non-Small Cell Lung Carcinoma Cells. Biomolecules 9.

Lee JH, Rangappa S, Mohan CD, Basappa, Sethi G, Lin ZX, et al. (2019c). Brusatol, a Nrf2 Inhibitor Targets STAT3 Signaling Cascade in Head and Neck Squamous Cell Carcinoma. Biomolecules 9: 550.

Li F, Shanmugam MK, Chen L, Chatterjee S, Basha J, Kumar AP, et al. (2013). Garcinol, a polyisoprenylated benzophenone modulates multiple proinflammatory signaling cascades leading to the suppression of growth and survival of head and neck carcinoma. Cancer prevention research 6: 843-854.

Liou CJ, Lai XY, Chen YL, Wang CL, Wei CH, \& Huang WC (2015). Ginkgolide C Suppresses Adipogenesis in 3T3-L1 Adipocytes via the AMPK Signaling Pathway. Evidence-based complementary and alternative medicine : eCAM 2015: 298635.

Liu G, Kuang S, Wu S, Jin W, \& Sun C (2016). A novel polysaccharide from Sargassum integerrimum induces apoptosis in A549 cells and prevents angiogensis in vitro and in vivo. Scientific reports 6: 26722.

Loh CY, Arya A, Naema AF, Wong WF, Sethi G, \& Looi CY (2019). Signal Transducer and Activator of Transcription (STATs) Proteins in Cancer and Inflammation: Functions and Therapeutic Implication. Frontiers in oncology 9: 48.

Mason GA (1949). Cancer of the lung; review of 1,000 cases. Lancet 2: 587-591.

Mohan CD, Rangappa S, Preetham HD, Chandra Nayaka S, Gupta VK, Basappa S, et al. (2020). Targeting STAT3 signaling pathway in cancer by agents derived from Mother Nature. Seminars in cancer biology.

Nakamura K, Mizuno Y, \& Kikuchi K (1996). Molecular cloning of a novel cytoplasmic protein tyrosine phosphatase PTP epsilon. Biochemical and biophysical research communications 218: 726-732.

Reck M, Heigener DF, Mok T, Soria JC, \& Rabe KF (2013). Management of non-small-cell lung cancer: recent developments. Lancet 382: 709-719.

Schindler C, Levy DE, \& Decker T (2007). JAK-STAT signaling: from interferons to cytokines. The Journal of biological chemistry 282: 20059-20063.

Shanmugam MK, Rajendran P, Li F, Kim C, Sikka S, Siveen KS, et al. (2015). Abrogation of STAT3 signaling cascade by zerumbone inhibits proliferation and induces apoptosis in renal cell carcinoma xenograft mouse model. Molecular carcinogenesis 54: 971-985.

Shanmugam MK, Warrier S, Kumar AP, Sethi G, \& Arfuso F (2017). Potential Role of Natural Compounds as Anti-Angiogenic Agents in Cancer. Current vascular pharmacology 15: 503-519.

Siegel RL, Miller KD, \& Jemal A (2015). Cancer statistics, 2015. CA: a cancer journal for clinicians 65: $5-29$.

Siveen KS, Ahn KS, Ong TH, Shanmugam MK, Li F, Yap WN, et al. (2014). Y-tocotrienol inhibits angiogenesis-dependent growth of human hepatocellular carcinoma through abrogation of AKT/mTOR pathway in an orthotopic mouse model. Oncotarget 5: 1897-1911.

Tanuma N, Nakamura K, \& Kikuchi K (1999). Distinct promoters control transmembrane and cytosolic protein tyrosine phosphatase epsilon expression during macrophage differentiation. European journal of biochemistry 259: 46-54.

Tanuma N, Nakamura K, Shima H, \& Kikuchi K (2000). Protein-tyrosine phosphatase PTPepsilon C inhibits Jak-STAT signaling and differentiation induced by interleukin-6 and leukemia inhibitory factor in M1 leukemia cells. The Journal of biological chemistry 275: 28216-28221.

Tanuma N, Shima H, Nakamura K, \& Kikuchi K (2001). Protein tyrosine phosphatase epsilonC selectively inhibits interleukin-6- and interleukin- 10-induced JAK-STAT signaling. Blood 98: 3030-3034. 
Tewari D, Nabavi SF, Nabavi SM, Sureda A, Farooqi AA, Atanasov AG, et al. (2018). Targeting activator protein 1 signaling pathway by bioactive natural agents: Possible therapeutic strategy for cancer prevention and intervention. Pharmacological research 128: 366-375.

Wang L, Syn NL, Subhash VV, Any Y, Thuya WL, Cheow ESH, et al. (2018). Pan-HDAC inhibition by panobinostat mediates chemosensitization to carboplatin in non-small cell lung cancer via attenuation of EGFR signaling. Cancer letters 417: 152-160.

Wang SW, \& Sun YM (2014). The IL-6/JAK/STAT3 pathway: potential therapeutic strategies in treating colorectal cancer (Review). International journal of oncology 44: 1032-1040.

Wong ALA, Hirpara JL, Pervaiz S, Eu JQ, Sethi G, \& Goh BC (2017). Do STAT3 inhibitors have potential in the future for cancer therapy? Expert opinion on investigational drugs 26: 883-887.

Xu L, Hu Z, Shen J, \& McQuillan PM (2015). Effects of Ginkgo biloba extract on cerebral oxygen and glucose metabolism in elderly patients with pre-existing cerebral ischemia. Complementary therapies in medicine 23: 220-225.

Yang MH, Jung SH, Chinnathambi A, Alahmadi TA, Alharbi SA, Sethi G, et al. (2019). Attenuation of STAT3 Signaling Cascade by Daidzin Can Enhance the Apoptotic Potential of Bortezomib against Multiple Myeloma. Biomolecules 10.

Yang MH, Lee JH, Ko JH, Jung SH, Sethi G, \& Ahn KS (2019). Brassinin Represses Invasive Potential of Lung Carcinoma Cells through Deactivation of PI3K/Akt/mTOR Signaling Cascade. Molecules 24: 1584.

Yang P, Allen MS, Aubry MC, Wampfler JA, Marks RS, Edell ES, et al. (2005). Clinical features of 5,628 primary lung cancer patients: experience at Mayo Clinic from 1997 to 2003. Chest 128: 452-462.

Yeh KY, Shou SS, Lin YX, Chen CC, Chiang CY, \& Yeh CY (2015). Effect of Ginkgo biloba extract on lipopolysaccharide-induced anhedonic depressive-like behavior in male rats. Phytotherapy research : PTR 29: 260-266.

Yu H, Pardoll D, \& Jove R (2009). STATs in cancer inflammation and immunity: a leading role for STAT3. Nature reviews Cancer 9: 798-809.

Zhang N, Zeng Y, Du W, Zhu J, Shen D, Liu Z, et al. (2016). The EGFR pathway is involved in the regulation of PD-L1 expression via the IL-6/JAK/STAT3 signaling pathway in EGFR-mutated non-small cell lung cancer. International journal of oncology 49: 1360-1368.

Zhang R, Han D, Li Z, Shen C, Zhang Y, Li J, et al. (2018). Ginkgolide C Alleviates Myocardial Ischemia/Reperfusion-Induced Inflammatory Injury via Inhibition of CD40-NF-kappaB Pathway. Frontiers in pharmacology 9: 109.

Zhou B, Shetye G, Yu Y, Santarsiero BD, Klein LL, Abad-Zapatero C, et al. (2020). Antimycobacterial Rufomycin Analogues from Streptomyces atratus Strain MJM3502. Journal of natural products 83: 657-667.

Zhu H, Chang LL, Yan FJ, Hu Y, Zeng CM, Zhou TY, et al. (2018). AKR1C1 Activates STAT3 to Promote the Metastasis of Non-Small Cell Lung Cancer. Theranostics 8:676-692.

\section{Figure legends}

Fig. 1. GGC alters STAT3 activation. (A) The structure of Ginkgolide C (GGC). (B) A549, H1299, and HEL 299 cells were treated with the indicated concentration of GGC for $24 \mathrm{~h}$ and viability was measured. (C) A549 cells were treated for indicated time intervals with GGC $30 \mu \mathrm{M}$ and western blotting was executed. (D)A549 cells were treated as described above in panel (C) and STAT3 levels were determined by EMSA. (E) A549 cells were treated with GGC $30 \mu \mathrm{M}$ for $12 \mathrm{~h}$ and STAT3 distribution was measured.(F) A549 cells were incubated with various concentrations of GGC and western blotting was executed. (G) Western blotting to analyze STAT5 (Tyr694/Tyr699) expression. (H) A549 cells were transfected with empty vector (pcDNA) or STAT3-C Flag pRc/CMV (300 ng) for $24 \mathrm{~h}$. Whole-cell extracts were prepared and Western 
blot was executed. (I) A549 cells were transfected with Empty vector (pcDNA) or STAT3-C Flag pRc/CMV were treated with or without of GGC for $24 \mathrm{~h}$ and cell viability was analyzed.

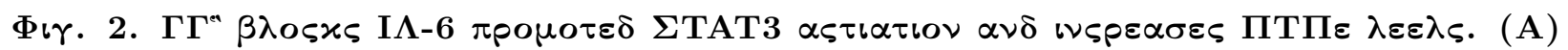
H1299 cells were treated with the indicated concentrations of GGC for $12 \mathrm{~h}$ and IL-6 $(20 \mathrm{ng} / \mathrm{ml})$ for 15 min. Thereafter western blot was executed. (B) H1299 cells were treated with GGC $30 \mu \mathrm{M}$ and stimulated with IL-6 as described above. Western blot was executed on the cell lysates. (C) A549 cells were treated as described above in panel (B) and western blotting was executed. (D) H1299 cells were transfected with STAT3 and treated with GGC for $12 \mathrm{~h}$. After stimulation with IL-6 (20 ng/ml) for $15 \mathrm{~min}$, luciferase assay was conducted. (E) A549 cells were exposed to pervanadate and $30 \mu \mathrm{M}$ of GGC for $12 \mathrm{~h}$ followed by western blotting. (F) A549 cells were treated with GGC for $12 \mathrm{~h}$ and western blot analysis was executed. (G) A549 cells were processed as elaborated above and RT-PCR was done. (H) A549 cells were transfected with PTPE-specific siRNA and scrambled RNA. After $24 \mathrm{~h}$, the cells were exposed to GGC $30 \mu \mathrm{M}$ for $12 \mathrm{~h}$. Lysates from the cells were determined by Western blot. (I) A549 cells were transfected and treated with as described above in panel $(\mathrm{H})$ and western blotting was executed.

Fig. 3. GGC promotes apoptosis and suppresses proliferation. (A) A549 cells were treated with GGC $30 \mu \mathrm{M}$ for $24 \mathrm{~h}$. and apoptosis was examined by Cell cycle Analysis, Annexin V, and TUNEL assays. H1299 cells were treated with GGC $30 \mu \mathrm{M}$. After $12 \mathrm{~h}$, cells were exposed to IL-6 $(20 \mathrm{ng} / \mathrm{ml})$ for $12 \mathrm{~h}$ and apoptosis was determined (B) A549 cell were treated with GGC for 24 h. H1299 cells were exposed to GGC. After $12 \mathrm{~h}$, IL-6 $(20 \mathrm{ng} / \mathrm{ml})$ was added in these cells for $12 \mathrm{~h}$. Thereafter western blotting was executed. (C) The cells were seeded in 16-well E-plates and treated with various concentration of GGC and IL-6 as elaborated above and proliferation assay was performed.

Fig. 4. GGC attenuates levels of tumorigenic proteins. (A) A549 cells were treated with GGC for $24 \mathrm{~h}$ and western blotting was executed.(B) H1299 cells were exposed to GGC $30 \mu \mathrm{M}$. After $12 \mathrm{~h}$, IL-6 $(20 \mathrm{ng} / \mathrm{ml})$ was added for $24 \mathrm{~h}$ and western notting was conducted.(C) A549 cells were treated with various concentration of GGC and RT-PCR was done for different genes. (D) H1299 cells were treated with GGC and IL-6 as elaborated above. (B) and RT-PCR was conducted. (E) A549 cell were transfected with PTPEspecific siRNA and scrambled RNA. After $48 \mathrm{~h}$, the cells were treated with GGC $30 \mu \mathrm{M}$ for $24 \mathrm{~h}$ and western blotting was executed. (F) A549 cells transfected with STAT3-C Flag pRc/CMV (300 ng) were treated with or without of GGC for $24 \mathrm{~h}$ and western blot analysis was conducted.

Fig. 5. Anti-tumor actions of GGC. (A) In vivo experimental design. (B) Necropsy photographs of mice carrying implanted tumors. (C) Tumor volume data. ${ }^{* *} \mathrm{p}<0.01$ compared the control. (D) Tumor weight was measured on Day 25. * $\mathrm{p}<0.05$ compared the control. (E) Body weight was calculated on the indicated days.

Fig. 6. GGC alters the levels of oncogenic markers in tissues. (A) GGC treated mice tissues were studied by immunohistochemical staining. Quantification data was represented as mean $\pm \mathrm{SD}$ on right panel. (B-E) Western blot data for different markers in whole cell extracts from mouse tissues. 
A.

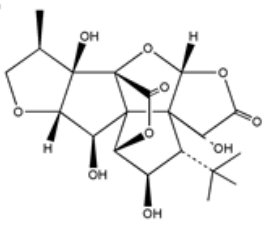

Ginkgolide C (GGC) [MW : 440]
B.

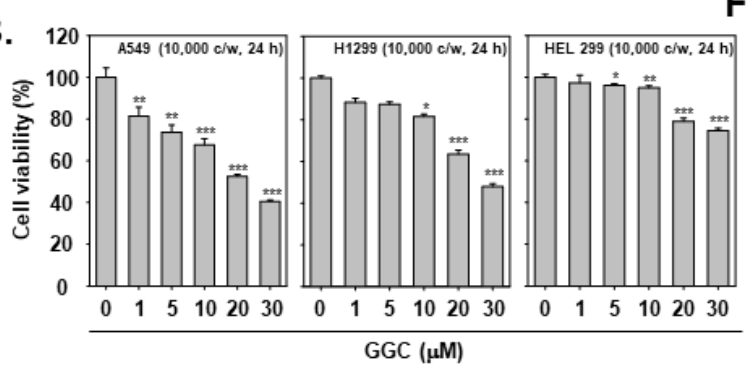

c.

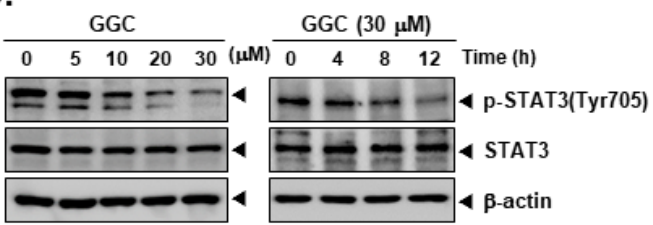

D.

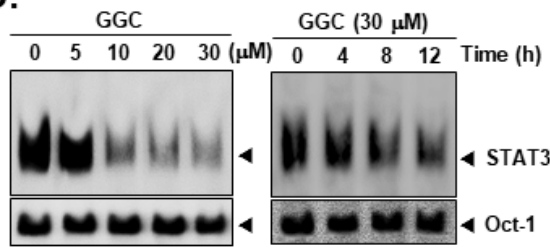

F.

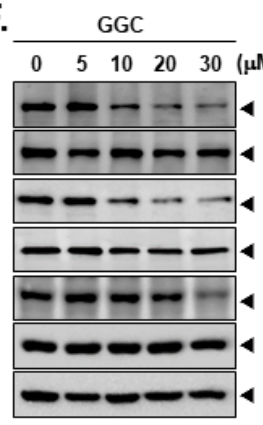

H.

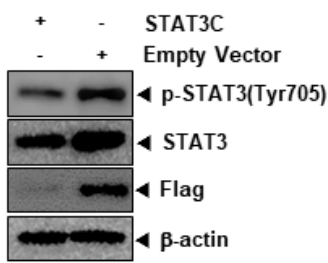

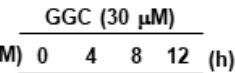

E.

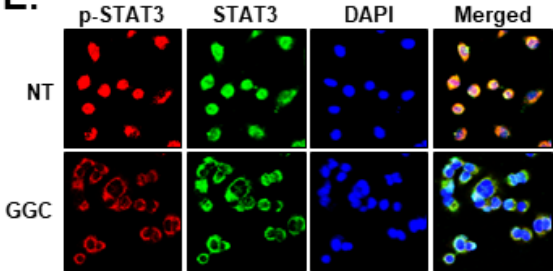

G.

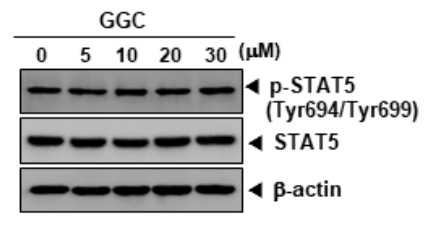

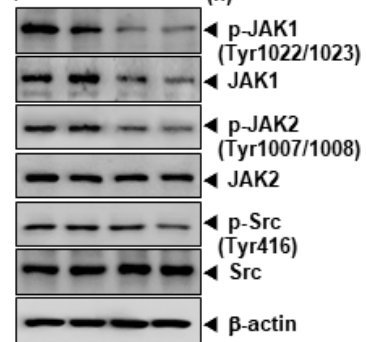

F1

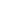

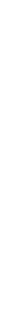


A.

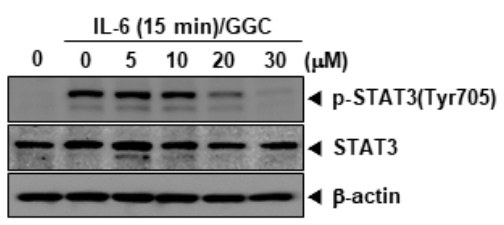

C.

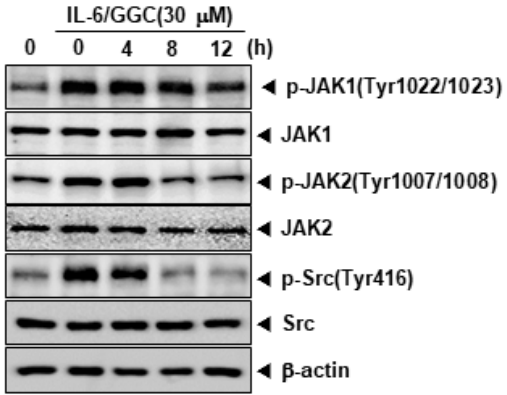

E.

-++++ GGC $(30 \mu \mathrm{M})$

$\begin{array}{llllll}0 & 0 & 1 & 5 & 10 & \text { Pervanadate }(\mu \mathrm{M})\end{array}$

- $= \pm \equiv$ - 2 -STAT3(Tyr705)

$-\infty-\infty$ STAT3

$-\infty-\infty$ \&-actin

G.

\begin{tabular}{ccccc}
\multicolumn{4}{c}{$\mathrm{GGC}$} & \\
\cline { 1 - 3 } & 10 & 20 & 30 & $(\mu \mathrm{M})$
\end{tabular}

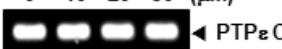

- -

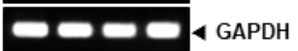

I.

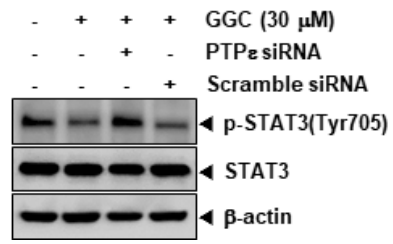

B.

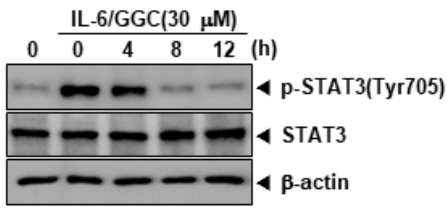

D.

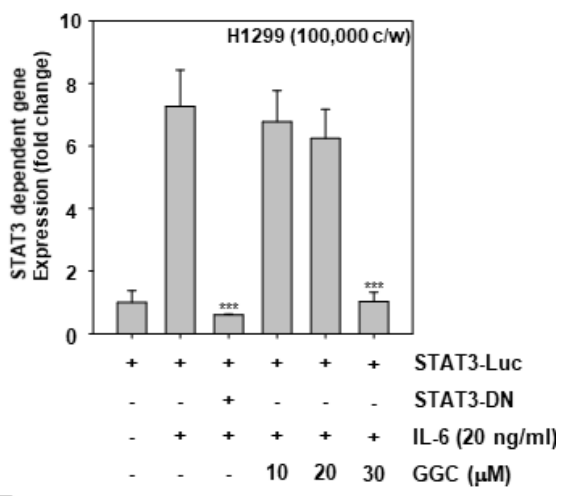

F.

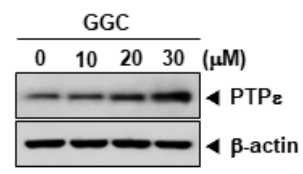

H.

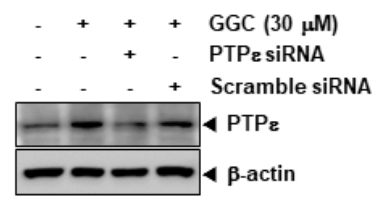


A.
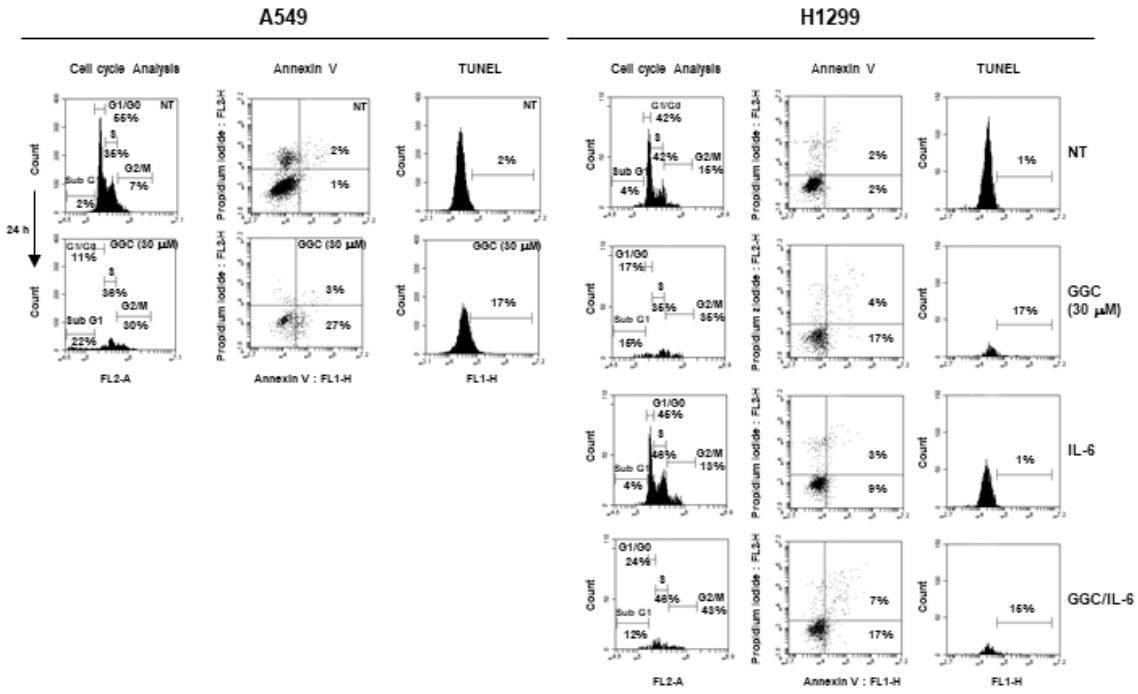

B.

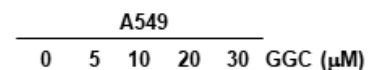

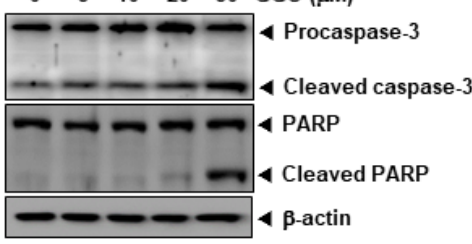

H1299

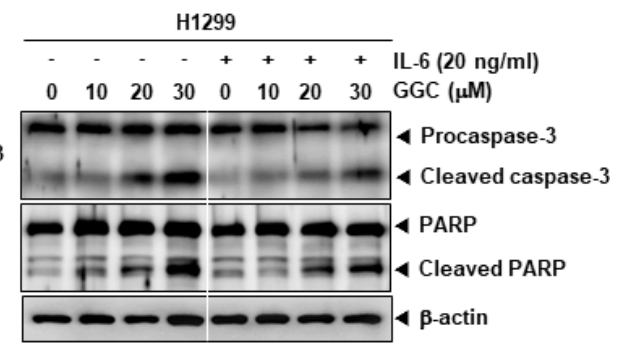

c.

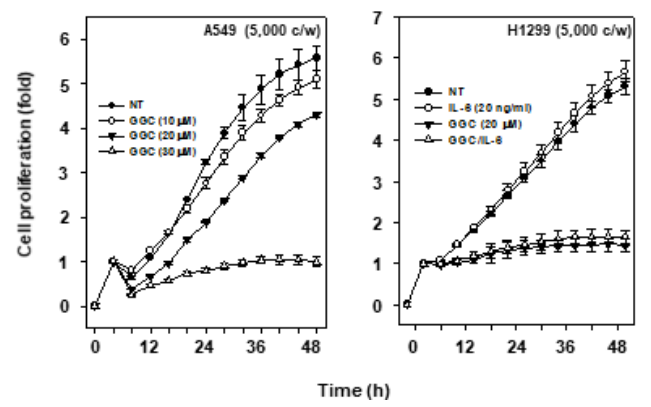


A.

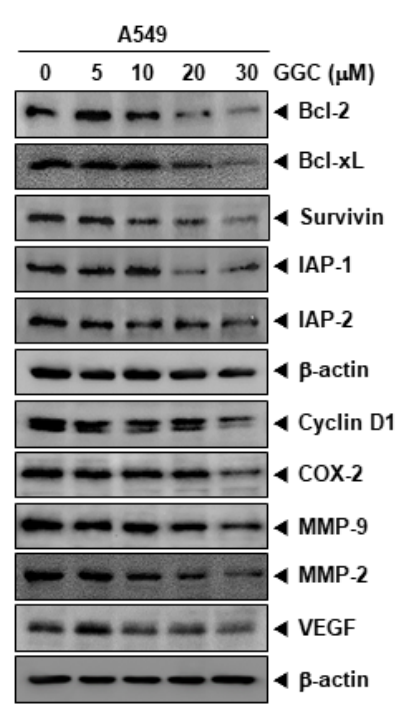

c.

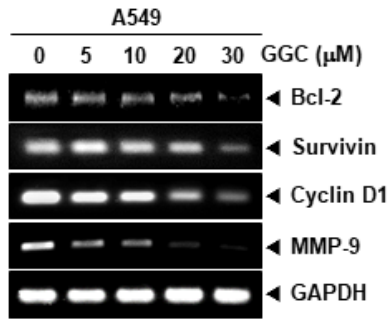

E.

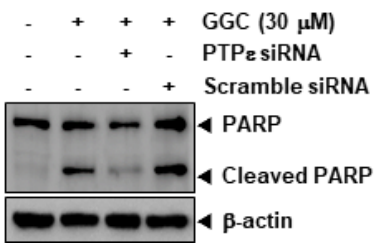

B.
D.

\begin{tabular}{|c|c|c|c|}
\hline \multicolumn{3}{|c|}{ H1299 } & \\
\hline 30 & 0 & 30 & $\mathrm{GGC}(\mu \mathrm{M})$ \\
\hline & & + & IL-6 (20 ng/ml) \\
\hline$=$ & ש & & $4 \mathrm{Bcl}-2$ \\
\hline - & $\sigma$ & & $4 \mathrm{Bcl}-\mathrm{xL}$ \\
\hline 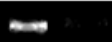 & 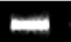 & & 4 Survivin \\
\hline$\sigma$ & 0 & & MMP-9 \\
\hline ש & $\sigma$ & & 4 GAPDH \\
\hline
\end{tabular}

F.

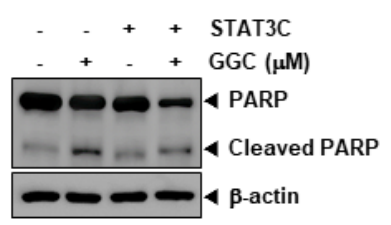


A.

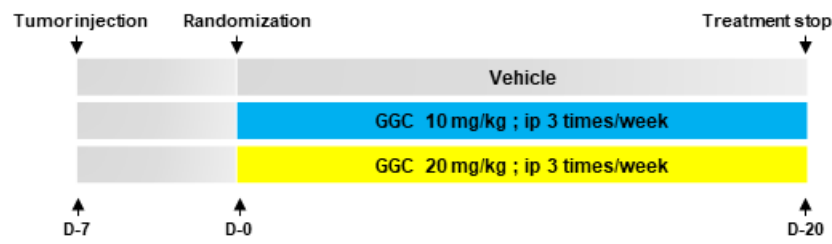

B.
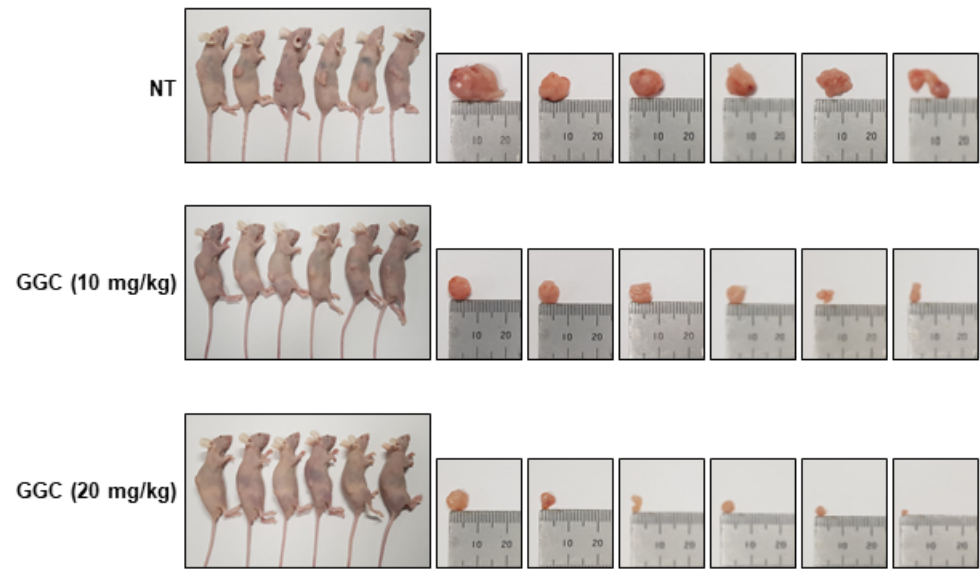

c.

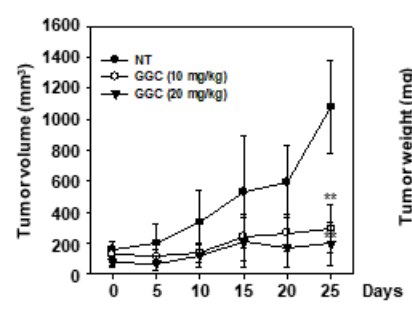

D.

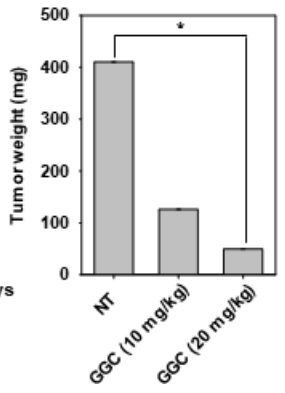

E.

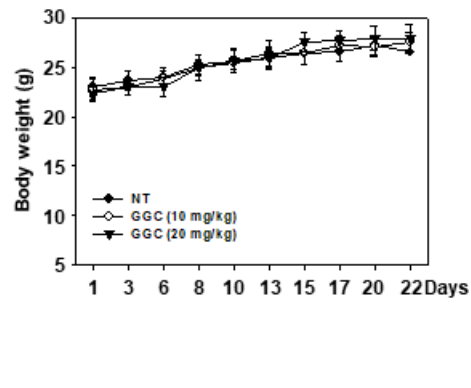


A.
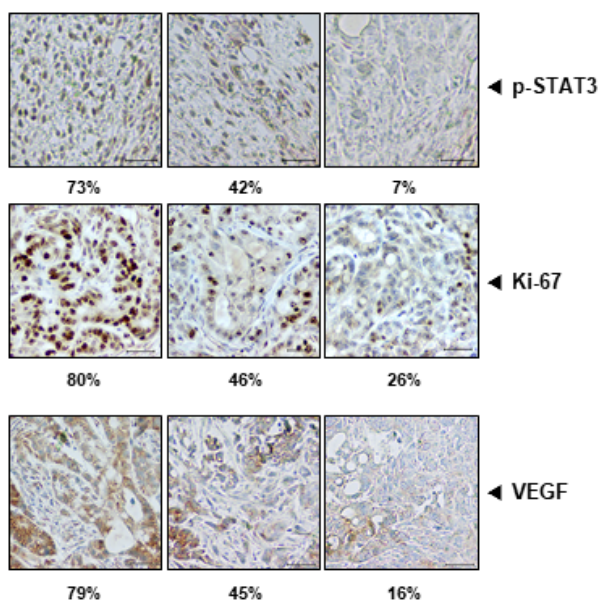

B.

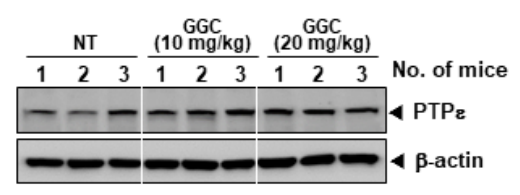

c.

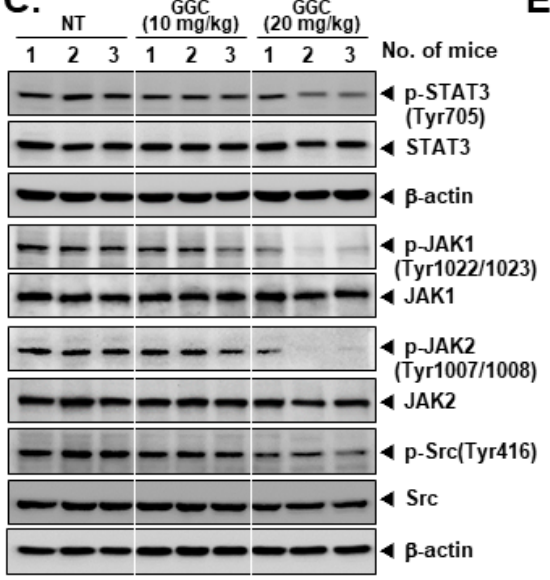
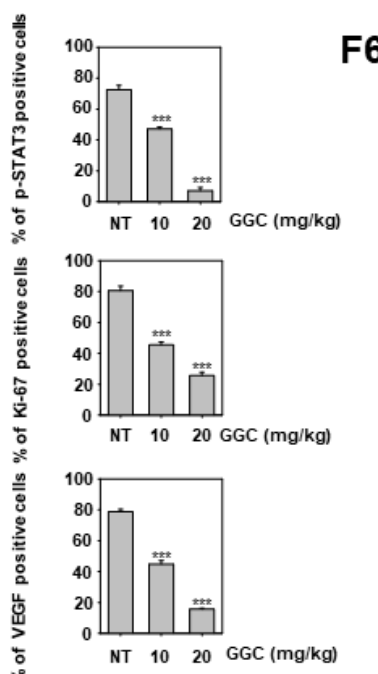

D.

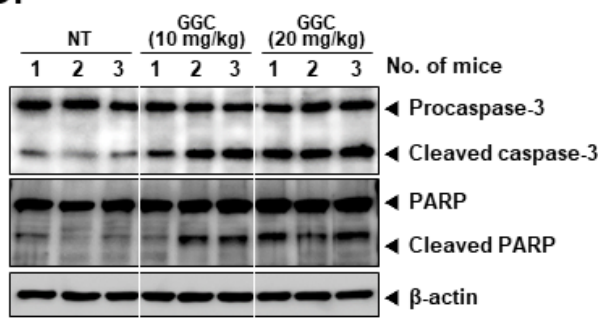

E.

\begin{tabular}{|c|c|}
\hline NT $\quad \frac{(10 \mathrm{mgC} / \mathrm{kg})}{(20 \mathrm{GgC} / \mathrm{kg})}$ & \\
\hline $\begin{array}{lllllll}2 & 3 & 1 & 2 & 3 & 1 & 2\end{array}$ & No. of \\
\hline$---\cdots$ & $4 \mathrm{Bcl}-2$ \\
\hline 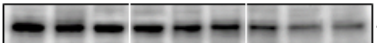 & $\int \mathrm{Bcl}-\mathrm{xL}$ \\
\hline$-\cdots--\cdots$ & 4 Survivin \\
\hline$------\cdots$ & 4 IAP-1 \\
\hline$----1=$ & 4 IAP-2 \\
\hline - $-\cdots-\cdots$ & । $\beta$-actin \\
\hline$--------\cdots$ & 1 Cyclin D1 \\
\hline---- & $4 \operatorname{cox}-2$ \\
\hline 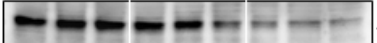 & $\checkmark \mathrm{VEGF}$ \\
\hline - $----\cdots$ & $4 \mathrm{MMF}$ \\
\hline - & \\
\hline
\end{tabular}

